\title{
Integer matrices obeying generalized incidence equations
}

\author{
Jennifer Wallis
}

\begin{abstract}
We consider integer matrices obeying certain generalizations of the incidence equations for $(v, k, \lambda)$-configurations and show that given certain other constraints, a constant multiple of the incidence matrix of a $(v, k, \lambda)$-configuration may be identified as the solution of the equation.
\end{abstract}

We define $(v, k, \lambda)$-configurations as usual (see [3]). If $B$ is the $(0,1)$ incidence matrix of a $(v, k, \lambda)$-configuration and if $A=b B$ where $b$ is a positive integer, then

$$
\left\{\begin{aligned}
A A^{T} & =b^{2}(k-\lambda) I+b^{2} \lambda J \\
A J & =b k J \\
\lambda(v-1) & =k(k-1),
\end{aligned}\right.
$$

with $J$ as usual the matrix with every element +1 , and $I$ the identity matrix. Ryser [2] proved a partial converse:

LEMMA 1. If $A$ is a $v \times v$ integer matrix satisfying equations (1) with $b=1$, then $A$ is the incidence matrix of a $(v, k, \lambda)$-configuration (and consequently has every entry 0 or 1 ).

One might conjecture, in view of the powerful theorems of Ryser [2] and Bridges and Ryser [1], that an integer matrix satisfying (1) would necessarily be $b$ times the incidence matrix of a $(v, k, \lambda)$-configuration. But the matrix

Received 24 August 1970. 


$$
A=\left[\begin{array}{rrrrrrr}
0 & 3 & 0 & 0 & 1 & 1 & 1 \\
-1 & 0 & 2 & 1 & 2 & 1 & 1 \\
2 & 0 & 0 & 0 & 2 & 2 & 0 \\
2 & 1 & 2 & 0 & 1 & -1 & 1 \\
1 & 0 & 0 & 1 & 0 & 1 & 3 \\
1 & 1 & 0 & 3 & 1 & 0 & 0 \\
1 & 1 & 2 & 1 & -1 & 2 & 0
\end{array}\right]
$$

satisfies (1) with $b=2, v=7, k=3$ and $\lambda=1$. So we need other conditions on the matrix $A$ before we can ensure that every element is 0 or $b$. We shall prove:

THEOREM 2. If $A$ is a $v \times v$ matrix of non-negative integers which satisfies ( 1 ), and if every entry of $A$ is less than or equal to $b$, then $A$ is $b$ times the incidence matrix of $a$ $(v, k, \lambda)$-configuration.

The corresponding result for non-positive $A$ and negative $b$ also holds.

By similar methods we shall obtain a result about more general equations :

THEOREM 3. Let $B$ be an integer matrix of order $v$ which satisfies

$$
\begin{aligned}
B B^{T} & =(p-q) I+q J \\
B J & =d_{J}
\end{aligned}
$$

where $p, q$ and $d$ are constants and $d>0$. Write $w$ and $z$ for the greatest and least elements of $B$ respectively, and $\omega=|\omega|$.

$I f$

$$
z \leq \frac{d}{v}=\delta \text { and } z \leq \frac{\omega d+p}{d+\omega v} \text {, }
$$

then $\delta$ is an integer, $p=d \delta=v \delta^{2}$, and $B=\delta J$.

\section{Proof of Theorem 2}

LEMMA 4. Let $B=\left(b_{i j}\right)$ of order $v$ be a matrix of non-negative integers such that $\sum_{j=1}^{v} b_{i j}^{2}=p, p$ a constant, for every $i$, and let 
$B J=d J, d$ a non-zero constant. If $b_{i j} \leq \frac{p}{d}$ for every $b_{i j}$, or if $b_{i j} \geq \frac{p}{d}$ for every non-zero $b_{i j}$, then every entry of $B$ is 0 or $\frac{p}{d}$.

$$
\text { Proof. } \begin{aligned}
\sum_{j=1}^{v} b_{i j}^{2} & =p \text { and } \sum_{j=1}^{v} b_{i j}=d \text {, so } \\
& d \sum_{j} b_{i j}^{2}-p \sum_{j} b_{i j}=d p-d p=0 ;
\end{aligned}
$$

that is

$$
\sum_{j} b_{i j}\left(d b_{i j}-p\right)=0
$$

From the data every term in this summation has the same sign, so every term is zero. So $b_{i j}=0$ or $\frac{p}{d}$.

COROLLARY 5. If there is a matrix $B$ satisfying the conditions of Lemma 4, then $d \mid p$.

Corresponding results may be obtained for matrices of non-positive integers.

Proof of Theorem 2. The matrix $A$ satisfies the conditions of Lemma 4 with $p=b^{2} k$ and $d=b k$. So every entry is 0 or $b \quad\left(b=\frac{p}{d}\right)$. Consider $B=b^{-1} A . \quad B$ is an integer matrix satisfying Lemma 1 , so it is the incidence matrix of a $(v, k, \lambda)$-configuration, and we have the result.

\section{Proof of Theorem 3}

Proof of Theorem 3. Clearly $p=\sum_{i} b_{i j}^{2}$ implies $p \geq 0 ;$ and $d>0$ implies $p>0$. Consider the class of matrices

$$
c_{\alpha}=B+\alpha J
$$

where $\alpha$ is an integer and $\alpha \geq \omega$. Every element of every member of this class is non-negative and 


$$
\begin{aligned}
C_{\alpha} C_{\alpha}^{T} & =(p-q) I+\left(\alpha^{2} v+2 \alpha d+q\right) J \\
C_{\alpha} J & =(d+\alpha v) J .
\end{aligned}
$$

Then using Lemma 4, if every non-zero element of $C_{\alpha}$ is less than or equal to $B$,

$$
\beta=\alpha+\frac{\alpha d+p}{d+\alpha v} \text {, }
$$

then every element is 0 or $B$.

We show that the conditions on $z$ imply that every element is $\leq \beta$. For

$$
z \leq \frac{\omega d+p}{d+\omega v}
$$

implies

$$
z(d+\omega v) \leq \omega d+p
$$

since $z \leq \frac{d}{v}$ we have

$$
z d+z \omega v+\gamma z v \leq \omega d+p+\gamma z v \leq \omega d+p+\gamma d
$$

for any integer $\gamma \geq 0$, so

$$
z \leq \frac{(\omega+\gamma) d+p}{d+(\omega+\gamma) v}
$$

This means (putting $\alpha=\omega+\gamma$ ) that for any admissable $\alpha$,

$$
z+\alpha \leq \alpha+\frac{\alpha d+p}{d+\alpha v}
$$

but $z+\alpha$ is the greatest element of $C_{\alpha}$. Therefore, any element of $C_{\alpha}$ is 0 or $\alpha+\frac{\alpha d+p}{d+\alpha v}$, so any element of $B$ is $-\alpha$ or $\frac{\alpha d+p}{d+\alpha v}$.

Corollary 5 tells us that

$$
A(\gamma)=\frac{(\omega+\gamma) d+p}{d+(\omega+\gamma) v}=\frac{d+p(\omega+\gamma)^{-1}}{d(\omega+\gamma)^{-1}+v}
$$

is integral for all integers $\gamma \geq 0$. Therefore $\lim A(\gamma)$ must be an integer, so $v \mid d$. Write $d=v \delta$ : 


$$
A(\gamma)=\frac{(\omega+\gamma) v \delta+p}{v \delta+(\omega+\gamma) v}
$$

so $v \mid p$. Write $p=\varepsilon v$ :

$$
A(\gamma)=\frac{(\omega+\gamma) \delta+\varepsilon}{\delta+(\omega+\gamma)}
$$

Choose $n$ any integer greater than $\delta+\omega$. Then

$$
A(n-\delta-\omega)=\frac{(n-\delta) \delta+\varepsilon}{n}
$$

so $n \mid\left(\varepsilon-\delta^{2}\right)$. But this is true for every large enough $n$; hence $\varepsilon=\delta^{2}$. That is

$$
\begin{aligned}
& d=v \delta \\
& p=v \delta^{2}
\end{aligned}
$$

so

$$
p=d \delta=v \delta^{2} \text {. }
$$

Then we have

$$
\frac{\alpha d+p}{d+\alpha v}=\frac{v \delta(\alpha+\delta)}{v(\delta+\alpha)}=\delta
$$

for any $\gamma$, so every element of $B$ is $-\alpha$ or $\delta$. Now the row sum of $B$ is $d=v \delta$ and the sum of the squares of the elements is $p=v \delta^{2}$; together these imply

$$
B=\delta J
$$

where $\delta=\frac{d}{v}$.

\section{References}

[1] W.G. Bridges and H.J. Ryser, "Combinatorial designs and related systems", J. Algebra 13 (1969), 432-446.

[2] H.J. Ryser, "Matrices with integer elements in combinatorial investigations", Amer. J. Math. 74 (1952), 769-773. 
[3] Herbert John Ryser, Combinatorial mathematics (The Carus Mathematical Monographs, No. 14. Math. Assoc. Amer., Buffalo, New York; John Wiley, New York, 1963).

University of Newcastle, New South Wales. 